\title{
ÉTUDE MORPHOLOGIQUE \\ DE LA MONTAGNE SAINTE-VICTOIRE
}

\author{
Adrien Billerey $\dagger$
}

Lorsque du sommet du Plateau du Cengle l'on embrasse dans tout son ensemble la chaîne de la Sainte-Victoire, s'opposent avec une netteté aveuglante, trois secteurs, aussi bien au point de vue topographique que structural et morphologique. Mieux encore, ces divisions tranchées se retrouvent dans l'avant-pays.

Saisir les causes profondes de contrastes aussi violents, entre les trois paysages morphologiques aux caractères si accusés, tel est, à nos yeux, l'un des problèmes fondamentaux.1

\section{Secteur Ouest}

L'Ouest oppose des plateaux monotones, vestiges grandioses d'une ancienne châne de montagne entièrement détraite, qui se déroulent au $\mathrm{N}$, à des crêtes et dépressions qui alternent vers le $\mathrm{S}$.

De Doudon au delà du barrage Zola, des Grands Creux aux abords du barrage du Bimont, des falaises occidentales de la Montagne Sainte-Victoire proprement dite, celles du Pas du Berger et de Beauroux, jusqu'au delà des Espinades à l'W, règnent sans partage, et sur de saisissantes épaisseurs, des brèches d'âges différents. Si, aux Espinades l'Infernet a buriné sur plus de $100 \mathrm{~m}$ des brèches vitrolliennes supérieures sans atteindre les brèches vitrolliennes inférieures sousjacentes, si le plateau du Bimont, plus élevé de $80 \mathrm{~m}$, est constitué par des brèches vitrolliennes inférieures que couronnent de minces brèches tertiaires, plus à l'E, dans le Plateau du Champ de Tir près de Roques Hautes, s'empilent des brèches vitrolliennes inférieures et vitrolliennes supérieures sur $190 \mathrm{~m}$. Enfin, constituant la façade occidentale de la chaîne, se superposent, en corniches imposantes et colorées, des brèches vitrolliennes inférieures, vitrolliennes supérieures, thapétiennes et bégudiennes, ces dernières emballées dans le plissement puis entrainées vers le sud par le décrochement.

Cette grandiose démolition d'une ancienne chaîne de montagne, dont il ne reste plus vers le $\mathrm{N}$ que le cœur sous la forme de calcaires séquaniens redressés à la verticale et tronqués à $370 \mathrm{~m}$, est riche de résonances. Cette masse énorme de brèches a été elle même arasée par une surface d'érosion que paracheva sans doute la transgression tortonienne dont les grès jaunes fossilifères sont les témoins sur le plateau du Bimont.

C'est donc une surface d'abrasion marine qui se déploie, parfaitement plane, sans aucune aspérité, au $\mathrm{N}$ des Grands Creux et que les grès jaunes discordants datent du Tortonien supérieur. Elle tranche des brèches qui, datées par R. Dughi, F. Sirugue et A. Billerey, prouvent l'existence de deux phases tectoniques, l'une de l'extrême fin du Crétacé, vitrollienne inférieure, l'autre du début même de l'Eocène, vitrollienne supérieure, donc d'âge laramien.

Or cette montagne vitrollienne s'est érigée plus au nord que la chaîne centrale actuelle. Entre les restes de la chaîne laramienne et le secteur central de la Montagne Sainte-Victoire, le décalage, qu'aucun auteur n'a signalé, et dont les conséquences tectoniques s'avèrent d'une importance capitale, et que souligne H. LiNigER, atteint une distance étonnante de $1600 \mathrm{~m}$.

De plus, la dénivellation de $80 \mathrm{~m}$ entre les Espinades et le plateau du Bimont, qu'accuse un talus méridien tout neuf, à peine ébréché par quelques vallons secs, prouve que la surface tortonienne a subi des cassures méridiennes et des décrochements horizontaux. Si la cassure post-tortonienne est spectaculaire entre les Espinades et les Grands Creux, le décrochement l'est plus encore sous le méridien de la ferme de Roques Hautes, souligné par une vallée rectiligne et le décalage des reliefs de part et d'autre des versants de celle-ci.

1 Voir Liniger, H.: Bau und Form der Ste-Victoire-Kette (Provence). Geographica Helvetica Nr. 2. Bern. 1962. 
Enfin, cette surface d'abrasion marine tortonienne a été non seulement cassée en blocs décrochés les uns des autres, ce qui suppose l'influence de voussoirs du socle, mais encore elle fut gauchie de l'W vers l'E, et de plus en plus gauchie à mesure que l'on se rapproche de la Sainte-Victoire. Nous avons suivi pas à pas les placages de grès jaunes tortoniens discordants sur les brèches. Si à l'W-SW ils se situent à $350 \mathrm{~m}$, à l'E-NE ils apparaissent jusqu'au dessus du Grand Vallon, à $410 \mathrm{~m}$. La surface d'abrasion sur le plateau du Bimont est donc inclinée vers l'W de $5 \%$.

Au delà vers l'E s'agit-il de la surface tortonienne, ou prétortonienne, rompue et portée à une altitude considérable, ou bien de reliefs prétortoniens de la chaîne que l'on pourrait au premier abord considérer comme des monadnocks, ou bien encore d'un relief plus ou moins rajeuni par des mouvements tectoniques récents? C'est ce que l'étude de la partie centrale nous permettra d'élucider.

C'est précisément l'érosion différentielle plio-quarternaire qui fut le processus majeur dans l'avant-pays occidental. La pénéplaine, ou la surface d'abrasion, a été disséquée vigoureusement par le réseau hydrographique de l'Infernet. La structure favorisa cette entaille: les ruisseaux s'adaptèrent aux failles longitudinales et aux décrochements méridiens. Et surtout, le contraste accusé entre la résistance des brèches et celle des marnes rouges a permis à l'érosion différentielle d'agir efficacement. Enfin, aujourd'hui encore, et avec une autre intensité au Quaternaire, les argiles rouges solifluent; des glissements massifs se sont produits. Ainsi, c'est à partir de la surface tortonienne que furent dégagées les crêtes longitudinales qui alternent avec des dépressions plus ou moins larges. C'est donc un véritable relief appalachien qui apparaît en bordure du plateau d'abrasion.

Au sud de ces collines appalachiennes, le creusement dans les marnes rouges de la dépression longitudinale du Tholonet et de la vallée transversale du Ruisseau de Bayeux mit en valeur et le Grand Cabri et le Plateau du Cengle. L'ampleur de l'érosion différentielle devient plus évidente encore en direction de l'Arc puisque trois séries de cuestas, thanétienne, vitrollienne, danienne, s'édifièrent soit à partir de la surface miocène, soit à partir de glacis plus récents descendus du Cengle.

Ainsi, dans ce secteur occidental, nous avons réussi à saisir les causes profondes des oppositions entre les deux paysages morphologiques. Au nord, les plateaux représentent la surface d'abrasion d'une part cassée en blocs décrochés, et d'autre part gauchie. Au sud, dans l'avant-pays, l'alternance des crêtes et des dépressions résulte de l'action de l'érosion différentielle aux dépens de la surface d'érosion. Si au nord, c'est la tectonique très récente post-pontienne qui individualise et nuance les plateaux d'abrasion, au sud, c'est la vigueur des processus des érosions quarternaires qui imprime au paysage sa marque fondamentale.

\section{Secteur Est}

A l'Est c'est la retombée périclinale d'un mont; son versant méridional curieusement buriné de très fréquents vallons secs, domine de bas glacis.

Au delà du décrochement de Delubre, dans le secteur oriental, les paysages morphologique diffèrent totalement des précédents, à la fois dans la montagne et dans l'avantpays. Mieux encore, les processus morphologiques les plus dynamiques, dans l'une et l'autre région, paraissent s'opposer, point par point, à ceux que nous venons de découvrir dans le secteur occidental.

A part la spectaculaire exception du Pic des Mouches, le point culminant de la chaîne, à 1010,6 m, la ligne de faite de ce secteur s'abaisse régulièrement vers l'E; et à $4,5 \mathrm{~km}$ du décrochement de Delubre, l'altitude n'est plus que de $575 \mathrm{~m}$. On passe donc progressivement de la montagne à la colline qui ferme à l'Orient le bassin de l'Arc supérieur. Renforce encore cette impression, la disparition définitive des falaises à l'E du Pic des Mouches. 


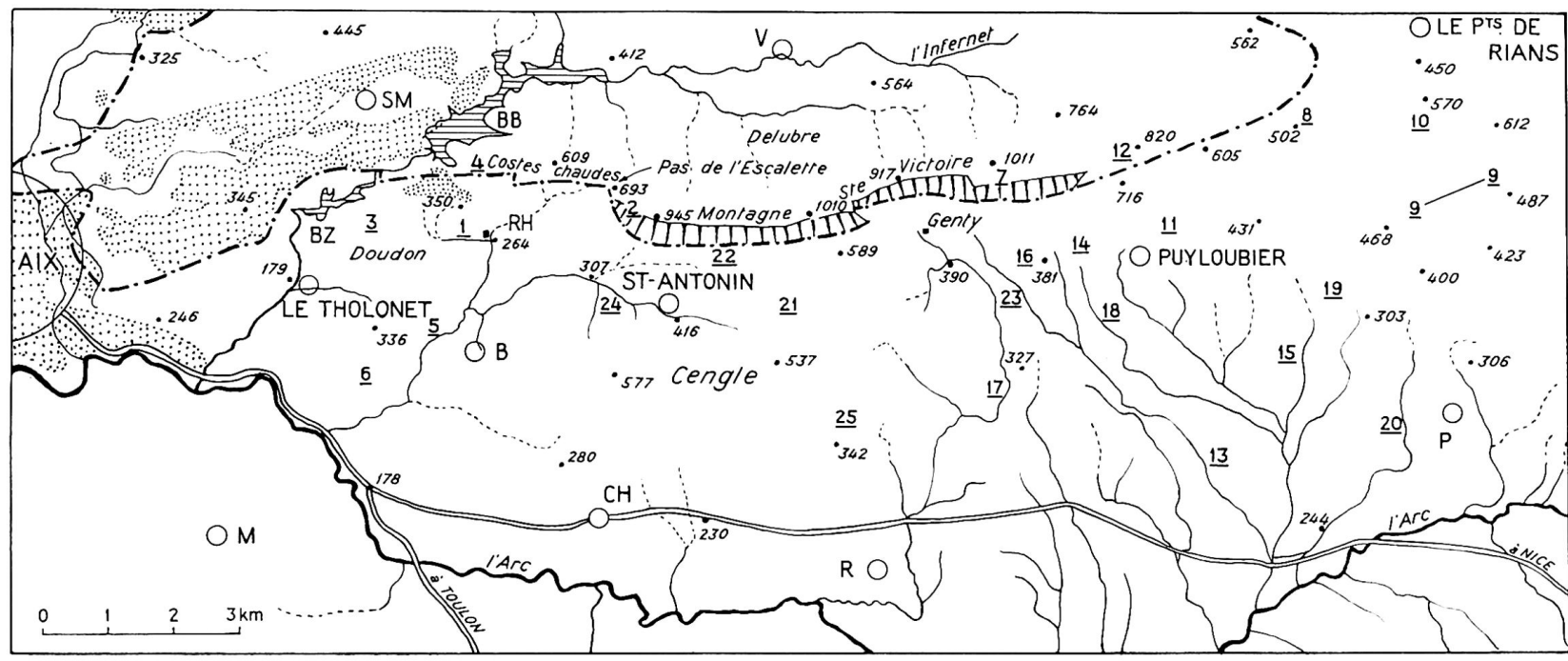

IIIT) falaise -.-.- terrain plissé

Tortonien marin

B Beaureceuil, BB barrage Bimont, BZ barrage Zola, CH Châteauneuf-le-Rouge, M Meyreuil, P Pourrières, R Rousset, RH Roques Hautes et Champ de Tir, SM St-Marc-Jaumegarde, V Vauvenargues. 1 Grands Creux, 2 Pas de Bergers, 3 les Espinades, 4 Grand Vallon, 5 ruisseau de Bayeux, 6 le Grand Cabri, 7 Pic des Mouches $(1010,6 \mathrm{~m}), 8$ la Palière, 9 Bois Communal de Pourrières, 10 la Dispute, 11 Trois Métronets (Mitronets), 12 Oratoire de Malivert (Mauvert), 13 ruisseau de St-Ser, 14 Clément, 15 St-Pont, 16 Bramelan, 17 Richeaume, 18 Miss d'Astier, 19 Callandre, 20 Beauvoisin, 21 Coquille, 22 dépression prémontagneuse, 23 Margaillan, 24 Vallée du Bayon, 25 l'Etang. 
Ce changement dans la structure prend tout son sens lorsque l'on met en évidencc le retrait vers le nord de tout ce secteur par rapport à la partie centrale. C'est donc désormais un Mont classique qui apparaît à sa place normale. Mais ce mont lui-même dégénère rapidement puisque se manifeste la retombée périclinale du pli qui se fond bientôt dans la bordure orientale du bassin.

En somme, le contraste ne peut pas être plus total avec l'W où de la surface d'abrasion, sans transition, surgissent les falaises vertigineuses de la chaîne occidentale. Le contraste est aussi éclatant au point de vue structural et tectonique car si l'W est la région des brèches sans montagne, l'E est le secteur de la montagne sans brèches. Ce qui paraît singulier c'est que ne se retrouve nulle trace des phases tectoniques: ni brèches bégudiennes, ni brèches vitrolliennes inférieures ou supérieures, ni brèches thanétiennes. Cependant, à Puyloubier une brèche préfuvélienne, au demeurant très peu puissante, dénote la présence d'un faible mouvement orogénique entre Valdonnien et Fuvélien.

Là aussi, montagne et avant pays présentent deux paysages étonnamment contrastés. L'observation minutieuse de la topographie montagneuse de cette terminaison périclinale du pli permet de déceler les traces de plusieurs surfaces d'érosion. La surface de la Bauxite est visible au Puits de Rians, à $450 \mathrm{~m}$; elle monte fortement vers la SainteVictoire, extraordinairement gauchie, ployée, cassée. Or cette pénéplaine du Crétacé moyen est recoupée par une ou deux surfaces moins bousculées, mais cependant déformées et surtout gondolées. Un décrochement, localisé au sud de Rians, où le plan de faille montre des stries horizontales et de la Bauxite écrasée, est nivelé par une surface d'érosion. La phase tectonique qui en découle ne peut être antérieure aux phases laramiennes; et la surface elle-même ne peut être antérieure aux pénéplanations oligocène ou miocène. Or il est curieux de noter que de part et d'autre de la surface sommitale se déploient deux surfaces d'érosion aux mêmes altitudes bien que les réseaux hydrographiques soient nettement différents. Au sud du Puits de Rians, à la Palière, une surface gauchie située entre 453 et $500 \mathrm{~m}$, recoupant la vieille surface à Bauxite, se relève vers le sud et surtout vers l'ouest. Au sud, un palier vraiment spectaculaire s'étale à 453/490 $\mathrm{m}$ dans le Bois Communal de Pourrières; il se relève également vers le faite et surtout vers l'W. D'une part il domine d'une centaine de mètres, par un talus raide, la zone des hauts glacis du bassin de l'Arc supérieur. D'autre part, une nette rupture de pente le sépare d'une série de croupes isolées qui forment l'axe topographique de la chaîne. Or ces croupes présentent toutes un sommet remarquablement plat. De plus, elles s'élèvent régulièrement de l'E à l'W: de $570 \mathrm{~m}$ à la Dispute, elles passent à $610 \mathrm{~m}$ aux Trois Métronets et atteignent plus de $820 \mathrm{~m}$ vers l'Oratoire de Mauvert.

Il résulte de ces observations que les deux surfaces qui flanquent la zone sommitale et qui se situent aux mêmes altitudes, et dont la méridionale domine les glacis quaternaires, sont des éléments de la surface tortonienne. La surface plus élevée, qui est plus gauchie, et qui ne peut être la pénéplaine à Bauxite, serait oligocène.

Ainsi, conséquences d'une importance primordiale, les reliefs prétortoniens n'auraient pas été entièrement arasés; de plus deux surfaces paraissent s'emboîter. Or cet emboîtement est particulièrement évident sur le flanc septentrional de la Sainte-Victoire au $\mathrm{N}$ de Vauvenargues; la surface inférieure est plus à l'W jalonnée par les grès tortoniens; la supérieure recoupe la surface à Bauxite très basculée.

Comme aucune phase tectonique n'a été mentionnée entre celle du début de l'Eocène et celle que nous avons identifiée au Plioquaternaire, il est vraisemblable de penser que l'édification de ce secteur périclinal est plioquaternaire. De fait, les vallons qui zèbrent le versant méridional paraissent n'avoir fonctionné que sous climat périglaciaire puisque nous avons surpris des grèses calcaires portlandiennes et kiméridgiennes au pied du talus.

Ainsi, dans ce secteur, se retrouve la pénéplaine du Crétacé moyen jalonnée par la Bauxite. Une esquisse de bordure montagneuse se forma avant le Fuvélien. Elle fut cassée et probablement reployée faiblement lors des phases bégudienne et laramiennes. 
Elle fut pénéplanée de nouveau à l'Oligocène. En fonction du niveau de la mer tortonienne s'emboîte alors une nouvelle surface. C'est donc au Plioquarternaire que cette terminaison périclinale de la chaîne prit son allure actuelle sous l'effet de rejeux tectoniques ou d'une phase indépendante. Ce serait ainsi un mont tout récent. Et le contraste s'avère bien fondamental entre l'W, où la montagne est la plus vieille et l'E où elle est la plus jeune. Si à l'W l'élément majeur de la morphologie, le plateau, résulte d'une abrasion marine, à l'E, l'élément majeur, le pli, est donné par la tectonique récente. Si à l'W les nuances morphologiques sont dues aux rejeux tectoniques plioquaternaires, à l'E elles sont déterminées par des pénéplanations diverses. Les facteurs déterminants de la morphologie sont donc curieusement inversés à l'W et à l'E dans le secteur de la montagne.

Il est encore plus étrange qu'ils le soient également dans l'avant-pays. L'avant-pays du secteur oriental se caractérise essentiellement par la présence d'un immense cône détritique dont l'axe est le Ruisseau de Saint-Ser et la base le cours supérieur de l'Arc, et dans lequel des glacis se sont superposés. S'y distinguent des glacis encroutés comme ceux de Puyloubier $\mathrm{N}$ et de Clément $\mathrm{E}$ et de St-Pons $\mathrm{N}$, des glacis d'accumulation comme ceux de Bramelan, de Clément $W$, de Puyloubier $S$, de Chantereine, un glacis pavé comme celui de Richeaume, des glacis d'érosion comme ceux de Mis d'Astier, de Calandre, de Beauvoisin. En dehors des glacis très élevés en bordure de la montagne, et des glacis très bas au voisinage de l'Arc, on peut distinguer trois étages: celui du haut glacis de Puyloubier N à $420 \mathrm{~m}$, celui du bas glacis de Saint-Pons à $290 \mathrm{~m}$ et celui des glacis intermédiaires comme Clément $\mathrm{E}$. Le matériel qui les recouvre le plus souvent provient du démantèlement superficiel de la chaîne sous climat froid. La présence de galets parfaitement arrondis parmi les pierrailles anguleuses, issus de la formation des Poudingues de la Galante, ne doit pas nous abuser. Comment donc ont-ils pu ici et ailleurs être interprêtés comme les témoins d'une pénéplaine? Ce sont les pulsations tectoniques qui sont à l'origine de cette accumulation; mais ce sont avant tous les processus d'érosion de climats froids qui en accrurent le volume, qui la mobilisèrent, qui la distribuèrent. Il ne nous semble pas en effet que les variations du niveau de base d'origine eustatique se soient fait sentir jusqu'aux sources des branches supérieures de l'Arc.

La morphologie de l'avant-pays résulte avant tout des effets des climats froids; l'élément fondamental est l'accumulation. L'examen de la partie centrale prend alors un intérêt saisissant. Clef de voûte du massif, elle semble détenir la clef des problèmes.

\section{Secteur Central}

Au centre s'érige à $1000 \mathrm{~m}$ une haute montagne calcaire, violemment dissymétrique et fortement décalée vers le Sud. Entre le plateau d'abrasion marine que se déroule à l'W, et la terminaison périclinale du pli qui s'incline vers l'E, le centre de la chaîne offre brusquement un aspect montagnard. Aspect montagnard insolite, celui d'une montagne en bordure d'un désert, si l'on ne retient que la façade et son piémont.

Eclatante de blancheur par la nature de ses calcaires, et par sa nudité, la chaîne se hausse subitement à $945 \mathrm{~m}$ à la Croix de Provence et est nettement décalée vers le $\mathrm{S}$. Si ce décalage par rapport aux restes de la montagne laramienne à l' $\mathrm{W}$, atteint $1500 \mathrm{~m}$, il n'est plus que de $500 \mathrm{~m}$ par rapport au secteur oriental. Ainsi cette partie centrale, flanc méridional renversé de l'anticlinal, s'est avancée obliquement par rapport à l'axe de la chaîne. Observation d'un intérêt prodigieux pour l'étude tectonique, et dont $\mathrm{H}$. LiNiger et nous-mêmes avons sondé les conséquences. Le problème fondamental, dès lors, est de savoir au cours de quelle phase tectonique s'est opéré ce décrochement oblique. Est-ce au cours de la grande phase tectonique bégudienne que matérialisent $200 \mathrm{~m}$ de brèches incorporées à la chaîne et renversées comme les assises de celle-ci? Est-ce au cours de la phase thanétienne dont les témoins sont des brèches de plus de $100 \mathrm{~m}$ de puissance qui, à l'avant de la chaîne, sont légèrement renversées? Est-ce au 
cours du rejeu plioquaternaire de la montagne que nous avons prouvé? Si décrochement et renversement des assises allaient de pair, la réponse serait simple, puisque dans l'avant-pays nous avons identifié l'accident à partir duquel les assises ne sont plus renversées, c'est-à-dire les terrains sparnaciens-yprésiens. C'est l'étude de la morphologie de ce secteur qui va nous permettre de proposer sinon une solution, du moins une hypothèse vraisemblable.

$\mathrm{Au}$ point de vue morphologique trois zones retiennent notre attention: la zone sommitale, le versant méridional et son piémont avec ses glacis étagés, enfin le Cengle.

a) Dans la zone sommitale deux aspects contrastés se succèdent d'W en E. Tout d'abord, la ligne de crête présente des dénivellations très accusées; elle n'est qu'une suite de sommets dissymétriques qu'isolent nettement de profonds vallons. Vers l'E par contre, une surface d'érosion curieusement plane et étonnamment subhorizontale tronque les assises suprajurassiques qui plongent très fortement vers le $\mathrm{N}$. De quelle surface s'agit-il?

Serait-ce la surface à Bauxite? Comme les calcaires suprajurassiques sont profondément recoupés par la surface, il s'ensuivrait qu'un plissement vigoureux aurait existé en cet endroit avant le Crétacé moyen. Or on ne retrouve aucun élément de sa destruction. Aucune brèche, pas plus qu'aucune trace de Bauxite n'ont été remarquées. De plus, il apparaît inconcevable qu'une surface aussi vieille ne soit pas fortement gauchie. Recoupant d'une part les vestiges orientaux de la surface à Bauxite elle ne peut que lui être postérieure; recoupant d'autre part des assises renversées elle ne peut être que postérieure aux assises renversées les plus récentes, c'est-à-dire aux assises sparnaciennesyprésiennes.

S'il ne s'agit pas de la surface la plus vieille, peut-être serait-ce la plus récente? La surface recoupe nettement les calcaires suprajurassiques; elle n'est pas gauchie; elle est subhorizontale. Mais alors si la surface tortonienne a été portée à cette altitude, le rejeu plioquarternaire deviendrait colossal, puisqu'il dépasserait, relativement, $640 \mathrm{~m}$. Dans ce cas, la chaîne aurait été édifiée presque totalement au Plioquaternaire. Il ne s'agirait plus d'un rejeu comme nous l'avions écrit dans une étude précédente mais d'une tectonique quarternaire indépendante. Nous hésitons devant une interprétation aussi audacieuse, bien qu'elle ne s'avère ni illogique, ni invraisemblable.

Reste une dernière hypothèse. La surface d'érosion sommitale, qui recoupe les assises renversées du Jurassique supérieur, devant être postérieure à l'assise renversée la plus récente, ne pourrait être que post-yprésienne, soit miocène ou oligocène. Mais cette éventualité conduit, dans l'état actuel de nos connaissances sur la Provence, à l'hypothèse précédente puisque dans la région aucune phase tectonique importante n'a été décelée entre l'Eocène et le Plioquaternaire. Cependant l'exhaussement de la chaîne pourrait n'être qu'en partie post-tortonien si la surface sommitale est oligocène et si l'emboîtement atteint vraisemblablement une hauteur plus forte qu'à l'E où il est de $100 \mathrm{~m}$.

L'observation de la façade et de son avant-pays nous apporte précisément à ce sujet quelques arguments.

b) La façade de la montagne Sainte-Victoire plonge, vertigineuse, de $415 \mathrm{~m}$ par une pente de $115 \%$ au dessus de Saint-Antonin et de $291,5 \mathrm{~m}$ par une pente de $130 \%$ au dessus de Coquille. En fait, deux falaises s'y superposent, entre lesquelles s'interpose un talus rocheux. La pente de celui-ci de $33 \mathrm{Gr}$ à l'W passe à $31 \mathrm{Gr}$ au centre et à 14 $\mathrm{Gr}$ à l'E. Or la falaise supérieure a subi les morsures intenses de l'érosion; parmi celles-ci nous intriguent ces niches imposantes aux parois polies, œuvre probable de tourbillons de neige glacée que déclenchaient sur ce relief les vents froids et eminemment turbulents du Quaternaire. Le talus à l'W de la Carrière est zébré de failles longitudinales inverses dont les miroirs apparaissent étonnamment frais. Enfin la seconde falaise qui tombe sur la dépression prémontagneuse présente dans sa partie occidentale des cannelures et des stries subverticales. Certes, il pourrait s'agir d'un plan de 
faille exhumé ou révélé. Mais cet accident oblique par rapport à la façade se prolonge au contact des brèches bégudiennes et du néocomien, en plein talus rocheux. C'est là qu'en certains points la fraîcheur du miroir est saisissante; il s'agit donc bien d'une faille qui aurait été pour le moins réactivée lors de la dernière phase tectonique.

Au demeurant, l'aspect du piémont renforce nettement cette impression d'un rejeu récent de la chaîne. Au delà de la dépression prémontagneuse, étroite et continue, une barre de brèches thanétiennes subverticales et même légèrement renversées court parallèlement à la façade du $\mathrm{N}$ de Margaillan $(401,4 \mathrm{~m}$ ) à l'W du décrochement de Delubre jusqu'au NW de Saint-Antonin. Or, vers l'E, cette barre se hausse en horsts à des altitudes anormales $(586,4 \mathrm{~m})$ par rapport aux reliefs encadrants. Sur le méridien de Saint-Antonin il est clair que ces crêtes se sont relevées depuis que les éboulis situés en aval les franchirent. Puis vers le $S$, jusqu'à la vallée du Bayon, descendent, s'étagent et s'emboîtent des glacis dont la base tranche les assises thanétiennes et sparnaciennesyprésiennes.

Le problème qui nous intéresse ici n'est pas de distinguer ces glacis d'après la nature de leur couverture; le problème essentiel est qu'une importante démolition de la chaîne tout au long du Quarternaire est attestée par la présence de glacis étagés. L'observation capitale est que les glacis les plus élevés ont été déformés par des mouvements tectoniques. Nous en avons fait la démonstration pour les glacis de la carrière, des Oiseaux et de Saint-Antonin. Ce dernier, le plus spectaculaire de tous, qui s'observe de la banlieue même d'Aix et qui surprend par sa subhorizontalité parfaite au dessus de la vallée du Bayon et en contrebas de la façade de Sainte-Victoire, mérite d'autant plus un examen qu'il a pû être interprété, depuis la parution de notre étude, comme un témoin d'une surface ludienne que l'on aurait prouvée sur le Cengle.

Non raccordé au talus rocheux de la façade dont la pente est de $33 \mathrm{Gr}$ et qui apparaît suspendu au dessus de la dépression prémontagneuse, ce glacis remarquablement plat contient sur plus de $20 \mathrm{~m}$ parfois des masses calcaires éboulées et écroulées d'un aspect cyclopéen reposant toujours sur des brèches. Au sein de cette couverture, et à $1000 \mathrm{~m}$ de la haute falaise de la chaîne, d'énormes blocs calcaires de $10 \mathrm{~m}$ sur 7 et 4 recouvrent des brèches et parfois des grèses périglaciaires. Or la base de cette couverture montre une allure synclinale. La couverture elle-même est ployée: au $\mathrm{N}$ elle plonge fortement depuis les brèches thanétiennes sur lesquelles elle passa; au centre, sa pente n'est plus que de $2,5 \mathrm{Gr}$; à l'extrémité méridionale du glacis cette couverture monte en direction du S par une pente de 20 à $25 \mathrm{Gr}$. Même sur un sol gelé des blocs de $10 \mathrm{~m}$ ne pouvaient progresser à contre-pente. Il est évident que la couverture du glacis, indubitablement quarternaire, dessine une inflection synclinale d'origine tectonique. Il ne nous était guère possible de préciser l'importance de ce rejeu. L'examen du Cengle nous permettra de proposer une hypothèse qui sera confrontée avec nos résultats antérieurs sur les secteurs orientaux et occidentaux.

c) J. Pouquet ayant observé des poudingues à la partie supérieure d'une colline couronnant le Cengle à l'E de la Ferme de l'Etang, les data du Ludien; il en inférait que le sommet du Cengle était constitué par une surface ludienne. Comme le sommet de la «butte» de Saint-Antonin, à quelques mètres près, se trouve à la même altitude, il devait s'ensuivre pour J. Pouquet que la «butte» de Saint-Antonin était une relique de la surface ludienne. Les poudingues dits ludiens sont indubitablement infra-lutétiens. De plus si la surface tortonienne elle-même apparaît gauchie, comme nous l'avons démontrée, comment serait-il possible que la «surface ludienne», beaucoup plus ancienne, fût restée imperturbablement horizontale au pied des falaises de la Sainte- $V$ ictoire? Enfin comment cette surface ludienne pourrait-elle recouper des formations quaternaires?

En fait, au sommet des collines du Cengle, sous le méridien de Saint-Antonin, nous avons recueilli, en présence de nombreux témoins, des plaques extrêmement minces d'un 
grès jaune assez semblable à celui des Bonfillons et absolument identique à l'un de ceux qu'offre la colline de La Tour, à l'E de Quinson. Malheureusement ces débris infimes de grès en partie décomposé et de plus dépourvu de fossiles peuvent prêter à contestation. Deux cas sont possibles:

D'une part si les analyses confirment l'âge tortonien de ces grès la surface sommitale du Cengle serait un témoin de la pénéplaine tortonienne. Le relèvement relatif du Cengle au Plioquaternaire serait alors de l'ordre de $300 \mathrm{~m}$, celui du secteur central de la Sainte-Victoire de $600 \mathrm{~m}$. Alors ou bien la surface d'érosion qui couronne la SainteVictoire au Baoû des Vêpres est elle aussi tortonienne ou bien elle représente une surface antérieure probablement oligocène d'après le paysage morphologique de la retombée périclinale orientale. Ou bien il ne s'agirait plus d'un rejeu tectonique mais d'une phase de plissement plioquaternaire particulièrement dynamique, ou bien la SainteVictoire, colline de $200 \mathrm{~m}$ de hauteur relative au Tortonien a subi un rejeu considérable ou les effets d'une phase de plissement plioquaternaire amortie.

Envisageons le second cas. Ce ne sont pas des grès tortoniens qui couronnent le Plateau du Cengle. Mais alors la seule conséquence est que la surface d'érosion sommitale ne peut être tortonienne. Dans ces conditions comme elle ne peut être du Crétacé moyen, elle serait oligocène.

Dans les deux hypothèses la Montagne Sainte-Victoire ne peut être considérée comme lutétienne comme l'affirma G. Corroy; il n'est pas question qu'elle soit un monadnock comme l'écrivit J. NiCOD; il est insoutenable que le glacis de Saint-Antonin soit une butte de la surface ludienne.

Le moment est donc venu de confronter l'ensemble de nos résultats. Si le processus morphologique majeur dans la partie septentrionale du secteur occidental était l'abrasion marine, dans le secteur oriental il est le mouvement tectonique plioquaternaire.

$\mathrm{Si}$ les processus secondaires à l'W étaient les rejeux tectoniques récents, à l'E c'étaient les pénéplanations du Crétacé moyen, de l'Oligocène, du Miocène.

Si dans l'avant-pays occidental le processus capital est l'érosion différentielle quaternaire, à l'E c'est une grandiose accumulation.

Dans le piémont du secteur central qui offre un étagement et un emboîtement remarquable de nombreux glacis, la multiplication des niveaux d'érosion et d'accumulation démontre de complexes interférences entre variations du niveau de base, pulsations tectoniques et oscillations climatiques. A notre avis, c'est l'exhaussement de l'avantpays central qui explique l'apparition de processus morphologiques contraires à l'W et à l'E, à l'W où parviennent les vagues d'érosion régressive, à l'E où elles ne parvinrent pas.

L'étude de la partie centrale de la chaîne résoud elle aussi, comme celle de son avant-pays, les contrastes et les oppositions radicales au point de vue morphologiques entre l'W et l'E. C'est l'avancée oblique de la partie centrale de la chaîne vers le $\mathrm{S}$ et son exhaussement qui expliquent la montée orientale périclinale de la chaîne au Plioquaternaire. Si à l'W la surface d'abrasion n'est que faiblement dénivelée et gauchie, c'est parce que c'est un voussoir du socle de direction oblique qui a rejoué au Quaternaire et que la conséquence en fut un décollement local de la couverture.

Ainsi donc à nos yeux, tout concorde pour faire de ce que nous appelions modestement, d'après le seul examen de l'avant-pays un rejeu plioquarternaire, une phase tectonique plioquaternaire réellement indépendante, quelle qu'en puisse paraître audacieuse l'idée.

La Bibliographie se trouve en: Billerey A. Un réjeu quaternaire de la Montagne de Sainte-Victoire. Extrait de «Méditerrannée», No. 2-3, 1960. 


\title{
ZUR MORPHOLOGIE DER SAINTE-VICTOIRE-KETTE
}

Der Verfasser versucht eine umfassende Synthese der Formung der bekannten Kette östlich Aix-en-Provence, basierend auf zum Teil noch unpublizierten Arbeiten. Die Antiklinale und ihr südliches Vorland zerfallen in drei völlig verschiedene Teile.

Westsektor. Falte und deren südliches Vorland sind auf $350 \mathrm{~m}$ Meereshöhe durch eine marine Abrasionsfläche im Prä-Torton eingeebnet. Die Gebirgsbildungen dieses Teils erfolgten im untern und obern Vitrolien (Danien und Montien), was durch mächtige Brekzienbänke erkennbar ist. Die Abtragungsfläche ist zerbrochen und steigt nach $E$ an; das südliche Vorland ist durch plioquartäre, selektive Erosion zerschnitten. Ostsektor. Von der Querstörung von Delubre nach $\mathrm{E}$ taucht die Kette axial von $1000 \mathrm{~m}$ bis ca. $450 \mathrm{~m}$ periklinal ab, die Absenz von Brekzien im Vorland deutet auf junge, plioquartäre Bildung des Sektors. Die prätortonische Fläche ist in der Kettenflanke bei Puyloubier und in der Scheitelregion bei Rian nachweisbar, aber gewellt und verstellt; lokal hat sie die Bauxitpeneplain der mittleren Kreidezeit abgedeckt. Im südlichen Vorland stellt man einen ausgedehnten Akkumulationskegel fest, der quartär bis rezente Terrassen aufweist, die auf sehr junge tektonische Bewegungen deuten.

Zentralabschnitt. Gemäß den Brekzien sind frühe orogenetische Phasen ins Bégudien und 'Thanétien zu setzen: Hebung und Überschiebung erfolgten im Plioquartär. Die leicht nach W fallende Gipfelflur liegt ca. 64o m höher als die Tortonfläche im W; sie wird als die stark gehobene Tortonfläche aufgefaßt. Die Falaise weist zwei verschiedene Regionen auf und ihre Piedmontfläche enthält diverse Terrassen, zum Teil quartär verborgen (Glacis von St. Antonin) Das im S anschließende Muldenstück des Cengle ist kein Rest einer «Ludienfläche», sondern ein Teil der Tortoneinebnung, allerdings um 200-300 m gehoben. Die jungen tektonischen Anomalien des Zentralsektors und die damit verbundene Hebung des Cengle-Abschnitts erklären die total verschiedene Formgebung von Kette und Vorland im W- und E-Sektor.

\section{KULTURGEOGRAPHISCHES AUS DEM NILDELTA}

\author{
Alfred Nawrath
}

Obwohl an der Nahtstelle des eurasischen und afrikanischen Kontinents gelegen und unfern des Suezkanals, der wichtigsten Wasserstraße der Alten Welt, gehört das Nildelta dennoch zu den wenig bekannten und wenig besuchten Landschaften rings ums Mittelmeer. Der Reisende unserer Tage, der die altarabische Weisheit, die Eile sei ein Kind des Satans, nicht wahrhaben will, fährt im dieselgetriebenen, klimatisierten weißen Expreßzug von Port Said oder Alexandrien direkt nach Kairo, der Stadt der Kalifen - sofern er nicht dorthin fliegt. Denn der Fliegende Teppich, von dem schon die größte Märchenerzählerin aller Zeiten, Schehrezâd, träumte, ist Wirklichkeit geworden!

Wer sich nicht begnügt mit dem, was heute ist, sondern gern wissen möchte, wie es im Delta einstens ausgesehen hat in historischer Zeit, ist, so paradox es klingen mag, fast ausschließlich angewiesen auf oberägyptische Quellen. Der Schlamm, welcher die alte Meeresbucht auffüllte und zu einer der fruchtbarsten, dichtest bevölkerten Landschaften unserer Erde machte, verändert das Delta ständig. Das Schwemmland hat ganze Städte weggeschluckt. Was ist z.B. noch übrig von Naukratis, das Thutmose gründete? Die Stadt war groß und reich und wußte zu leben: Herodot rühmt die Schönheit ihrer Hetären. Von Naukratis führte eine Wasserstraße zur alten Reichshauptstadt Memphis. Sie brauchte keinen Vergleich zu scheuen mit den großen Überlandstraßen der Perser, die von Susa bis Sardes führten. Herodot erwähnt, daß man zur Zeit der Hochflut nicht unbedingt auf die Kanalroute angewiesen war; man konnte den Weg abkürzen, indem man über die überschwemmten Felder hinweg dicht am Fuß der Pyramiden entlangsegelte.

Glücklicherweise war das Delta zur Pharaonenzeit beliebtes Erholungsgebiet, und zum Glück hielten die Notablen des Alten, Mittleren und Neuen Reichs ihre dortigen 